\title{
Importancia de la simulación Phet en la enseñanza y el aprendizaje de fracciones equivalentes*
}

\section{Importance of Phet simulation in teaching and learning equivalent fractions}

\section{Importância da simulação Phet no ensino e $o$ aprendizagem de frações equivalentes}

\author{
Jorge Enrique Díaz Pinzón** \\ Fecha de recepción: 20 de julio de 2016 \\ Fecha de evaluación: 15 de marzo de 2017 \\ Fecha de aceptación: 8 de mayo de 2017 \\ Disponible en línea: 18 de mayo de 2017 \\ DOI: http://dx.doi.org/10.18359/reds.2011
}

Cómo citar este artículo:

Díaz Pinzón, J.E. (2017). Importancia de la simulación Phet en la enseñanza y el aprendizaje de fracciones equivalentes. Revista Educación y Desarollo Social, 11(1), 48-63. DOI: org/10.18359/reds.2011 enseñanza y aprendizaje de fracciones equivalentes (2016).

** Magíster en Gestión de la Tecnología Educativa, de la Universidad de Santander (UDES). Docente de Matemáticas, de la Institución Educativa General Santander, Soacha, Colombia. Correo electrónico: jorgediaz333@gmail.com-jediazp@unal.edu.co 


\section{Resumen}

En el presente artículo de investigación se utilizó un material didáctico multimedia (Phet) que integra los componentes de tecnología y pedagogía con el fin de apoyar el proceso de enseñanza y aprendizaje de matemáticas para el octavo grado de la Institución Educativa General Santander, de Soacha, Cundinamarca. El objetivo es determinar si la aplicación de simulaciones Phet mejora la enseñanza y el aprendizaje de fracciones equivalentes, de acuerdo con los contenidos curriculares de la asignatura Matemáticas. Esta investigación es de tipo experimental de enfoque cuantitativo, con Pretest y Postest, para cada grupo de comparación. La población de estudio estuvo constituida por 40 estudiantes de octavo grado: el grupo 1 estuvo conformado por 20 estudiantes ( 10 hombres y 10 mujeres) y el grupo 2, por 11 hombres y 9 mujeres. El instrumento que se usó fue la prueba escrita y prueba virtual (simulador Phet), para ambos grupos. Se concluyó por inferencia estadística que el rendimiento académico para el grupo 1 no mejoró significativamente al utilizar el simulador Phet en la enseñanza de las fracciones equivalentes. Para el grupo 2 el rendimiento académico de los estudiantes mejoró significativamente al utilizar el simulador Phet para la enseñanza de las fracciones equivalentes.

Palabra claves: Simuladores matemáticos, tecnologías de la información, estrategia didáctica, inferencia estadística.

\section{Abstract}

In this research article, a multimedia didactic material (Phet) was used. That material integrates components of technology and pedagogy to support the teaching and learning process of mathematics for the eighth grade of the. Educational institution General Santander in Soacha, Cundinamarca. The objective is to determine whether the application of Phet Simulations improves teaching and learning of equivalent fractions, according to the curricular contents of the subject Mathematics This research is an experimental type, of a quantitative approach, with pre-test and post-test for each comparison group. The study population consisted of 40 students from eighth grade: group 1 was composed of 20 students (10 men and 10 women) and group 2 by 11 men and 9 women. The Instrument used was Written Test and Virtual Test (Phet Simulator) for both groups. It was concluded from a statistical inference that the academic performance for group 1 did not improve significantly when using the simulator PHET in teaching equivalent fractions. For group 2, the academic performance of the students considerably improved when using the simulator Phet in teaching equivalent fractions.

Keywords: Mathematical simulation, information technology, teaching strategy, statistical inference.

\section{Resumo}

No presente artigo de pesquisa foi utilizado um material didático multimídia (Phet) que integra os componentes de tecnologia e pedagogia com o fim de apoiar o processo de ensino e aprendizagem de matemática para o oitavo grau da Instituição Educativa General Santander, de Soacha, Cundinamarca. O objetivo é determinar se a aplicação de simulações Phet melhora o ensino e a aprendizagem de frações equivalentes, de acordo com o conteúdo curricular do curso de Matemática. Esta pesquisa é do tipo experimental de abordagem quantitativa, com Pré-teste e Pós-teste para cada grupo de comparação. A população de estudo foi composta por 40 alunos de oitavo grau: o grupo 1, teve conformado por 20 estudantes (10 homens e 10 mulheres) e o grupo 2, por 11 homens e 9 mulheres. O instrumento utilizado foi à prova escrita e prova virtual (simulador Phet) para ambos os grupos. Conclui-se, por inferência estatística que o desempenho académico no grupo 1 não melhorou significativamente quando ao utilizar o simulador Phet no ensino das fracções equivalentes. Para o grupo 2 o desempenho acadêmico dos alunos melhorou significativamente quando o uso do simulador Phet para o ensino das frações equivalentes.

Palavras-chave: simuladores matemáticos, tecnologia da informação, estratégia didáctica, inferência estatística. 


\section{Introducción}

La internet es considerada en la actualidad como uno de los más importantes fenómenos del comienzo del siglo XXI. $\mathrm{Su}$ desarrollo, unido al extraordinario progreso en todas las técnicas de comunicación e información, puede ser comparado con el nacimiento de la escritura o de la imprenta.

El desarrollo de las nuevas tecnologías de la información y la comunicación relacionadas con la internet ha abierto nuevos espacios en el ámbito educativo. El diseño de sitios web que integran múltiples aplicaciones multimedia está dotando a los docentes en general de nuevos ambientes de aprendizaje donde predomina la interactividad y el rol del profesor innovador, lo que facilita al estudiante la construcción de su propio conocimiento.

No obstante, se debe tener claro que si bien la tecnología educativa es un elemento importante para mejorar los procesos de enseñanza-aprendizaje, esta mejora no depende solamente de la utilización de un software educativo, sino además de su adecuada integración curricular, es decir, del entorno educativo diseñado por el docente.

La problemática radica en que al estudiante se le dificulta representar situaciones diversas que involucren los 50 diferentes usos de las fracciones con base en disímiles contextos. Por tal motivo, el diseño y la adaptación de simulación de la plataforma Phet permite el estudio de las fracciones equivalentes en términos de encontrar fracciones semejantes usando números y gráficas, enlazar o emparejar fracciones con diferentes tipos de gráficas; esto contribuye y facilita el aprendizaje del concepto de fracciones equivalentes.

Mediante el uso de simulaciones Phet como una ilustración animada, se encontró que es más fácil comunicarse efectivamente con los estudiantes. Los Phet muestran procesos dinámicos y estos pueden ser lentos abajo, acelerados o en pausa, dependiendo del concepto que se muestre; lo invisible se hace visible, y múltiples representaciones están vinculadas. Por último, los sims se ajustan fácilmente por el instructor durante el debate. Estas características hacen a menudo sims más eficaces para el aprendizaje y más práctico utilizar los dibujos estáticos o demostraciones en vivo. Phet está diseñado para ayudar a los estudiantes a desarrollar habilidades de investigación científica mediante la exploración de las relaciones de causa y efecto.

Los instructores pueden facilitar la consulta de toda la clase mediante la creación de un escenario en la simulación y pidiendo a los estudiantes que predigan, manipulado, el efecto de variables. En estos salones de clase, los estudiantes a menudo de forma espontánea preguntan muchos más, y más profundo, preguntas. Es común que los estudiantes pidan una serie de "qué pasaría si" preguntas y dirigir el uso de la tarjeta SIM de los profesores. 
En cuanto a los simuladores, estos "Son objetos de aprendizaje que mediante un programa de software intentan modelar parte de una réplica de los fenómenos de la realidad y su propósito es que el usuario construya conocimiento a partir del trabajo exploratorio, la inferencia y el aprendizaje por descubrimiento" (Peña y Alemán, 2013, p. 1). También, según Fatela (2012), "Los simuladores digitales son aplicaciones interactivas que simulan situaciones de experimentos físicos reales o que ilustran temas matemáticos" (p. 1).

Los simuladores son programas que representan un modelo o entorno dinámico, que a través de gráficos o animaciones facilitan al estudiante la visión de lo que ocurre en el entorno que se está simulando, de forma que modificando de manera interactiva las características del entorno puede comprender mejor lo que sucede en el entorno que está intentando conocer. Dada la actualización de la tecnología, siempre debemos estar en busca de nuevos simuladores que sean más efectivos e interesantes. (Ortega, 2001, p. 276)

Respecto a lo anterior, Bagur (2011) menciona lo siguiente:

En lo personal, creo que para que estos simuladores tengan todo el éxito que pueden lograr es necesario, primero, que el docente identifique muy bien los elementos del tema a enseñar; que trate el tema por medio de algunos ejemplos y luego use a los simuladores como medios de práctica o evaluación. (p. 2)

Los simuladores matemáticos ofrecen variedades de temas en esta área del conocimiento, contienen una explicación muy didáctica, divertida, entretenida y sobre todo con la mayor claridad posible, con muchos ejemplos de aplicación a la vida cotidiana para que el usuario le saque el mejor provecho a este tipo de herramientas que abundan en internet. (Durán, 2012, p. 1)

De acuerdo con Muñoz (2012), "Es una página de gran valor didáctico con muy entretenidas e interactivas simulaciones gratuitas apoyados en investigaciones del proyecto Phet de la Universidad de Colorado" (p. 1). Además agrega que "Inicialmente el proyecto se centró en simulaciones de física y fue nombrado como Physics Education Technology Project, o Phet. Cuando lanzaron simulaciones de química, biología, ciencias de la tierra, matemáticas y otras áreas, decidieron mantener el nombre de Phet" (p. 1).

Dada la importancia de utilizar simuladores como herramienta de aprendizaje, varios autores hacen mención al tema. Galicia (2005), en su tesis Las aulas virtuales en el proceso enseñanza aprendizaje en el nivel medio, ciclo básico, sector privado del área urbana del municipio de Jalapa, departamento de Jalapa, destaca el progreso de la ciencia y la tecnología, y el uso del computador como una herramienta necesaria en todos los ámbitos sociales. 
Añade Arbeláez (2010), en su tesis Mundos virtuales para la educación en salud, simulación y aprendizaje en Open Simulator, menciona que el uso de simulación y de videojuegos se ha transferido con fuerza en la industria, las prácticas militares y la medicina, y esto ha generado en los últimos años una poderosa herramienta de formación. En lo relacionado con la medicina se han desarrollado simuladores de equipos y dispositivos de procedimientos de alto riesgo y simuladores para fortificar procesos educativos en la salud. Estas instrucciones se han introducido a otras áreas como la educación.

De igual forma, Pósito (2012) menciona que los modernos avances tecnológicos actuales han logrado grandes impactos en la educación, y se han ampliado así los escenarios educativos, con lo cual se han brindado medios de comunicación y soporte de materiales para proporcionar la interacción entre las personas. Además, se planteó el objetivo de ofrecer soluciones tecnológicas y pedagógicas al problema del diseño de habilidades de aprendizaje para aprender ciencias naturales en los nuevos contextos educativos.

Contribuye a esto García (2012) en la tesis titulada Promover en el aula estrategias de aprendizaje para elevar el nivel escolar de los estudiantes de tercero primaria en el área de Matemá-

52 tica, al cavilar sobre las insuficiencias en el aprendizaje de las matemáticas por diferentes factores y despunta la importancia de utilizar estrategias de aprendizaje para perfeccionar el nivel académico del estudiante.

La metodología empleada en el estudio es de tipo experimental-cuantitativa, con un grupo control y un grupo experimental (prueba con el simulador Phet). La población de estudio estuvo constituido por 40 estudiantes de octavo grado de educación secundaria de la Institución Educativa General Santander de Soacha-Cundinamarca.

\section{Metodología}

El proyecto se realizó mediante un tipo de investigación experimental, que es el siguiente:

Aquella que permite como mayor seguridad establecer relaciones de causa a efecto. Pues presenta una visión general y aproximada del objeto de estudio, además de contar con una investigación de tipo cuantitativo. Se ha escogido una metodología de tipo cuantitativa el diseño de la investigación cuantitativa establece un método experimental habitual del conjunto de las normas científicas. (Monje, 2011, p. 105)

Regularmente, a estos experimentos se los nombra ciencia verdadera y manejan medios matemáticos y estadísticos cotidianos para evaluar los resultados de modo concluyente.

Todos los experimentos cuantitativos utilizan un formato estándar, con algunas pequeñas diferencias interdis- 
ciplinarias para generar una hipótesis que será probada o desmentida. Esta hipótesis debe ser demostrable por medios matemáticos y estadísticos y constituye la base alrededor de la cual se diseña todo el experimento. (Shuttleworth, 2008, p. 1)

La asignación aleatoria de un grupo de estudio es primordial y debe contener un grupo de control, siempre que sea posible. Un diseño cuantitativo solo debe manejar una variable a la vez, de lo contrario el análisis estadístico puede ser muy complejo y dispuesto a interrogantes.

Este tipo de metodología permite al investigador diseñar ambientes de aprendizaje, lo cual implica una mayor comprensión de sus elementos (estudiante, objeto matemático, contexto, artefacto o recurso tecnológico, situación-problema, actividad estructurada, etc.), así como anticipar cómo estos van a funcionar conjuntamente para promover el aprendizaje.

\section{[...]}

Los estudios de diseño permiten explicar por qué el diseño funciona y sugieren modos en que puede ser adaptado a nuevas circunstancias, de acuerdo a problemas y situaciones emergentes. Pensamos que esta metodología es pertinente con la investigación a desarrollar, ya que permitirá obtener argumentaciones basadas en la evidencia procedente de contextos naturales, de abordar cuestiones teóricas sobre la naturaleza del aprendizaje en contexto y de producir resultados a partir de evaluación formativa. (Cobb et al., 2003, citados por Huapaya,2012, pp. 56)

Una de las particularidades de esta metodología es que permite asumir el rol de investigadores y docentes, de esta manera se puede variar y tomar decisiones en los experimentos prescritos. La segunda característica es de intervención, pues proponemos mejorar el aprendizaje de los elementos de la función cuadrática, a través de prácticas de simulación de situaciones-problema mediadas por el Phet, buscando que el estudiante haga uso de las diversas representaciones de esta herramienta matemática.

\section{Población}

La población, según Balestrini (2006), se define como "cualquier conjunto de elementos de la que se quiere conocer o investigar alguna de sus características" (p. 126). Este proyecto se centra en estudiantes de la jornada de la tarde, específicamente del grado 801 cuyas edades oscilan entre los 12 y 15 años.

\section{Muestra}

La muestra está representada por los estudiantes de octavo grado y se utilizó la siguiente fórmula para calcular su tamaño:

$$
n=\frac{Z^{2} \sigma^{2} N}{e^{2}(N-1)+Z^{2} \sigma^{2}}
$$


Donde:

$\mathrm{n}=$ el tamaño de la muestra.

$\mathrm{N}$ = tamaño de la población.

$\sigma=$ Desviación estándar de la población en la que, generalmente cuando no se tiene su valor, suele utilizarse un valor constante de 0,5.

$\mathrm{Z}=$ Valor obtenido mediante niveles de confianza. Es un valor constante que si no se tiene su valor, se lo toma en relación con el $95 \%$ de confianza que equivale a 1,96 (como más usual) o en relación con el $99 \%$ de confianza que equivale 2,58 , valor que queda a criterio del investigador.

e = Límite aceptable de error muestral en el que, generalmente cuando no se tiene su valor, suele utilizarse un valor que varía entre el $1 \%(0,01)$ y el $9 \%$ $(0,09)$, valor que queda a criterio del encuestador.

Se tiene $\mathrm{N}=43$, para el $99 \%$ de confianza $Z=2,58$, y como no se poseen los demás valores se tomará $\sigma=$ y e $=0,05$.

Reemplazando los valores en la fórmula se obtiene:

$$
\begin{gathered}
n=\frac{N^{2} \sigma^{2} Z}{(N-1) e^{2}+\sigma^{2} Z^{2}} \\
n=\frac{43-0,5^{2}-2,58^{2}}{(43-1)( \pm 0,05)^{2}+0,5^{2}-2,58^{2}}=\frac{71.55}{1.76}=
\end{gathered}
$$

$$
=40.4=40
$$

\section{Muestreo aleatorio simple}

Según Huillcañahui (2010), "Es aquel en cada elemento de la población tiene la misma probabilidad de ser seleccionado para integrar la muestra, cada uno de los elementos de la muestra, se selecciona aleatoriamente uno por uno". (p. 7)

Para esta investigación se realizó un muestreo sin reemplazo en este tipo. Según Huillcañahui (2010), "no se devuelven los elementos extraídos a la población hasta que no se hayan extraído todos los elementos de la población que conforman la muestra". (p. 9)

Con la muestra de 40 estudiantes, se realizó un sorteo con 20 papeletas que decían grupo experimental y 20 que decían grupo control, y se sacaron uno a uno, tantos como indicara el tamaño de la muestra, en este caso 40 . Posteriormente el grupo control pasó a ser el grupo experimental y el grupo experimental pasó a ser el grupo control.

Esto se hizo con la finalidad de diferenciar los grupos; además, se realizó una prueba pretest y postest (tratamiento con el simulador Phet) a cada uno de los grupos.

\section{Hipótesis}

La hipótesis del presente trabajo de investigación se diseña como una relación causal y se enuncia de la siguiente forma: 
Hipótesis Alterna (Ha): en la aplicación del uso de simulaciones Phet existen diferencias significativas promedio o la media de calificaciones entre los grupos 1 y 2 en el pretest y el postest, con el tema relacionado con fracciones equivalentes en los estudiantes de octavo grado.

Hipótesis Nula (Ho): en la aplicación del uso de simulaciones Phet no existen diferencias el promedio o la media de calificaciones entre los grupos 1 y 2 en el pretest y postest, en cuanto al tema relacionado con fracciones equivalentes en los estudiantes de octavo grado (ver tabla 1).

Tabla 1. Comprobar si existen diferencias en el pretest y postest, grupos 1 y 2

\begin{tabular}{|l|l|l|l|}
\hline Grupo 1 & $\mathrm{O} 1$ & $\mathrm{X}$ & $\mathrm{O} 2$ \\
\hline Grupo 2 & $\mathrm{O} 3$ & $\mathrm{X}$ & $\mathrm{O} 4$ \\
\hline
\end{tabular}

Fuente: elaboración propia.

O1: Pretest G1

O3: Pretest G2

$\mathrm{X}$ : Tratamiento con el simulador Phet

O2: Postest G1

O4: Postest G2

\section{Identificación y clasificación de las variables}

Variable independiente: estimar el uso del simulador Phet para la comprensión del concepto fracciones equivalentes en los estudiantes de grado octavo de la Institución Educativa General Santander. Instrumento: prueba escrita y prueba virtual. Variable dependiente: estrategia didáctica para la adquisición del aprendizaje del concepto de la función cuadrática en los estudiantes de noveno grado de la Institución Educativa General Santander. Instrumento: plan de estudios octavo grado.

\section{Estrategia didáctica aplicada}

La estrategia implicó dos etapas:

- Instruccional: etapa para preparary motivar al estudiante en qué y cómo iba a aprender. En esta fase se explicó en qué consistía la experiencia, y se indicaron además el sistema de evaluación, las actividades por desarrollar en el computador y el manejo del software de simulaciones (Muñoz, 2012).

- Postinstruccional: etapa para fomentar el desarrollo de un aprendizaje significativo, en la cual se introdujo el contenido temático de fracciones equivalentes. Se desarrollaron ejercicios, en un principio con ejemplos visualizados a través del software de simulaciones (Muñoz, 2012) (ver figura 1), y luego el estudiante desarrolló diez ejercicios para certificar los resultados a través del mismo programa. 


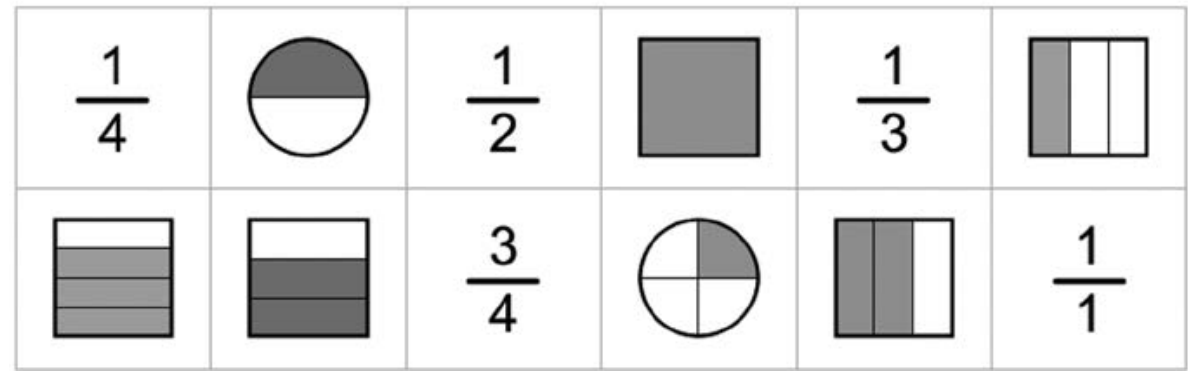

Figura 1. Ejemplo visualizado del simulador Phet

\section{Instrumentos}

Las herramientas aplicadas durante la investigación fueron las siguientes: prueba de conocimientos pretest. Evaluación con diez preguntas aplicada como prueba de conocimientos sobre fracciones equivalentes al inicio de la actividad didáctica y como prueba final al culminar esta, una prueba postest en el simulador (Phet) también con diez ejercicios.

\section{Prueba estadística}

El valor de significancia de la prueba es de $\sigma=0.05$ ( $5 \%$ ); si es mayor se acepta la hipótesis nula y si es menor se rechaza la hipótesis nula. Para una variable aleatoria (numérica), se aplicará la prueba t de student, para diferencia de medias en muestras relacionadas. Es una prueba estadística para evaluar si dos grupos difieren entre sí de manera significativa respecto de sus medias. Se utilizará el Software SPSS v 23.0.

\section{Resultados}

En las tablas 2 y 3 se pueden observar los resultados del pretest y postest para los grupos 1 y 2 .

Tabla 2. Resultados del grupo 1 en el Pretest y Postest

\begin{tabular}{|c|c|c|}
\hline Estudiante & Pretest & Postest \\
\hline 1 & 7 & 9,1 \\
\hline 2 & 8 & 10 \\
\hline 3 & 10 & 8,3 \\
\hline 4 & 10 & 8,3 \\
\hline 5 & 8 & 10 \\
\hline 6 & 8 & 7,5 \\
\hline 7 & 10 & 9,1 \\
\hline 8 & 9 & 9,1 \\
\hline 9 & 9 & 10 \\
\hline 10 & 10 & 10 \\
\hline 11 & 7 & 10 \\
\hline 12 & 9 & 9,1 \\
\hline
\end{tabular}




\begin{tabular}{|c|c|c|}
\hline Estudiante & Pretest & Postest \\
\hline 13 & 8 & 9,1 \\
\hline 14 & 7 & 10 \\
\hline 15 & 10 & 8,3 \\
\hline 16 & 5 & 10 \\
\hline 17 & 9 & 10 \\
\hline 18 & 9 & 8,3 \\
\hline 19 & 10 & 10 \\
\hline 20 & 8 & 10 \\
\hline
\end{tabular}

Tabla 3. Resultados grupo 2 en el pretest y postest

\begin{tabular}{|c|c|c|}
\hline Estudiante & Pretest & Postest \\
\hline 1 & 8 & 9,1 \\
\hline 2 & 5 & 9,1 \\
\hline 3 & 9 & 10 \\
\hline 4 & 8 & 10 \\
\hline 5 & 9 & 10 \\
\hline 6 & 10 & 9,1 \\
\hline 7 & 9 & 10 \\
\hline 8 & 10 & 10 \\
\hline 9 & 7 & 10 \\
\hline 10 & 10 & 10 \\
\hline 11 & 8 & 10 \\
\hline 12 & 10 & 9,1 \\
\hline 13 & 10 & 10 \\
\hline 14 & 9 & 10 \\
\hline 15 & 10 & 10 \\
\hline 16 & 7 & 10 \\
\hline 17 & 9 & 8,3 \\
\hline 18 & 9 & 10 \\
\hline 19 & 10 & 9,1 \\
\hline 20 & 9 & 10 \\
\hline
\end{tabular}

Fuente: elaboración propia.

\section{Interpretación de los resultados}

Se debe corroborar que la variable aleatoria en ambos grupos se distribuye normalmente. Para ello se utiliza la prueba de Kolmogorov-Smirnov, cuando las muestras son grandes ( de 30 individuos) o la prueba de Chapiro Wilk, cuando el tamaño de la muestra es menor de 30 individuos). El criterio para evaluar si la (VA) se distribuye normalmente es:

P- valor $=>\alpha$ aceptar $\mathrm{Ho}=$ los datos provienen de una distribución normal.

P- Valor $<\alpha$ aceptar $\mathrm{Hl}=$ los datos no provienen de una distribución normal.

Igualdad de varianza (pruebe de Levende). Se debe corroborar la igualdad de varianza entre los grupos.

P-valor $=>\alpha$ aceptar $\mathrm{Ho}=$ las varianzas son iguales.

P- Valor $=<\alpha$ aceptar $\mathrm{Hl}=$ existe diferencia significativa entre las varianzas.

- Calcular el p-valor de la prueba: t student muestras independientes.

\section{Normalidad}

Kolmogorov-Smirnov: muestras grandes (> de 30 individuos).

Chapiro Wilk: muestras pequeñas $(<\mathrm{de}$ 30 individuos).

Criterio para determinar la normalidad: 
P- valor $=>\alpha$ aceptar $\mathrm{Ho}=$ los datos provienen de una distribución normal.

P- Valor $<\alpha$ aceptar $\mathrm{Hl}=$ los datos no provienen de una distribución normal.

Al no comportarse normalmente la variable de calificación para el pretest y postest en el grupo 1 , se procedió a realizar la prueba de Anderson-Darling:

Es utilizada para probar si un conjunto de datos muéstrales provienen de una población con una distribución de probabilidad continua específica (por lo general la distribución normal). La prueba de Anderson-Darling se basa en la comparación de la distribución de probabilidades acumulada empírica (resultado de los datos) con la distribución de probabilidades acumulada teórica (definida en H0). (López, 2011, p. 2)

El valor estadístico al grupo $1\left(\mathrm{~A}^{2}=\right.$ $-40,93)$ es menor que el valor crítico $\left(\mathrm{A}^{2}\right.$ crítico $\left.=0.752\right)$; por lo tanto, no se rechaza la hipótesis nula. Por esta razón, los datos observados en el postest tienen una naturaleza de distribución normal.

Tabla 4. Prueba de normalidad grupo 1. Pretest-postest

\begin{tabular}{|l|c|c|c|c|c|c|c|}
\hline \multirow{2}{*}{} & \multirow{2}{*}{ Grupo 1 } & \multicolumn{3}{|c|}{ Kolmogorov-Smirnov } & \multicolumn{3}{c|}{ Shapiro-Wilk } \\
\cline { 3 - 8 } & & Estadístico & gl & Sig. & Estadístico & gl & Sig. \\
\hline Pretest & &, 180 & 20 &, 089 &, 879 & 20 &, 017 \\
\hline Postest & &, 304 & 20 &, 000 &, 795 & 20 &, 001 \\
\hline
\end{tabular}

Fuente: elaboración propia

Tabla 5. Prueba de normalidad grupo 2. Pretest-postest

\begin{tabular}{|l|l|c|c|c|c|c|c|}
\hline \multirow{2}{*}{} & \multirow{2}{*}{ Grupo 2 } & \multicolumn{3}{|c|}{ Kolmogorov-Smirnov } & \multicolumn{3}{c|}{ Shapiro-Wilk } \\
\cline { 4 - 9 } & & Estadístico & gl & Sig. & Estadístico & gl & Sig. \\
\hline Pretest & &, 260 & 20 &, 001 &, 821 & 20 &, 002 \\
\cline { 4 - 9 } & &, 427 & 20 &, 000 &, 634 & 20 &, 000 \\
\hline
\end{tabular}

Fuente: elaboración propia. 
Al no comportarse normalmente la variable calificación, para el pretest y postest en el grupo 2, se procedió a realizar la prueba de Anderson-Darling:

Es utilizada para probar si un conjunto de datos muestrales provienen de una población con distribución de probabilidad continua específica (por lo general la distribución normal). La prueba de Anderson-Darling se basa en la comparación de la distribución de probabilidades acumulada empírica (resultado de los datos) con la distribución de probabilidades acumulada teórica (definida en H0). (López, 2011, p. 2)

El valor estadístico al grupo 2, Pretest $\left(A^{2}=-40,6\right)$ y postest $\left(A^{2}=-41\right)$, es menor que el valor crítico $\left(\mathrm{A}^{2} \mathrm{Critico}=0.752\right)$; por lo tanto, no se rechaza la hipótesis nula. Así pues, los datos observados en el postest tienen una naturaleza de distribución normal.

La variable "calificación" ahora se comporta normalmente para los grupos 1 y 2 , en pretest y Postest, y realizados los ajustes se aplicó la t student para muestras relacionadas.

Prueba t student (decisión estadística).

El criterio para decidir es:

Si la probabilidad obtenida de P-valor $\leq \alpha$, rechazo Ho (se acepta $\mathrm{Ha}$ ).

Si la Probabilidad obtenida de P-valor > $\alpha$, no rechazo Ho (se acepta Ho).

De esta manera el P-valor pretest-postest del grupo $1=, 074>\alpha=0,05$.

Tabla 6. Prueba T para muestras relacionadas grupo

\begin{tabular}{|l|l|c|c|c|}
\hline \multirow{2}{*}{} & \multicolumn{3}{|c|}{ Prueba t para la igualdad de medias } \\
\cline { 3 - 5 } & $\mathbf{t}$ & gl & Sig. (bilateral) \\
\hline Par 1 & $\begin{array}{l}\text { Pretest } \\
\text { Postest }\end{array}$ & $-1,892$ & 19 &, 074 \\
\hline
\end{tabular}

Fuente: elaboración propia.

Tabla 7. Prueba T para muestras relacionadas grupo 2

\begin{tabular}{|l|l|c|c|c|}
\hline \multirow{2}{*}{} & \multicolumn{3}{|c|}{ Prueba t para la igualdad de medias } \\
\cline { 3 - 5 } & $\mathrm{t}$ & gl & Sig. (bilateral) \\
\hline Par 1 & $\begin{array}{l}\text { Pretest } \\
\text { Postest }\end{array}$ &, 3185 & 19 &, 01 \\
\hline
\end{tabular}

Fuente: elaboración propia. 
El P-valor pretest-postest del grupo 2 = $, 01<\alpha=0,05$

\section{Discusión de resultados}

Contribuir al mejoramiento del aprendizaje de los conceptos fundamentales de las matemáticas —en este caso las fracciones equivalentes, mediante la innovación pedagógica - es una labor de los docentes que imparten esta área de conocimiento, con el propósito de obtener los mejores resultados y motivar a los estudiantes; por lo tanto, es relevante conocer estrategias y herramientas que acompañen el aprendizaje de las matemáticas.

En la presente investigación se logró identificar que el simulador Phet aplicable a las fracciones equivalentes, y dadas las características de su uso en línea o descargar al computador para su uso, ofrece una simulación gratuita e interactiva.

El simulador aplicado en la presente investigación se encuentra en el área de simulaciones de matemáticas, simuladores de fraccionarios, del sitio web https://phet.colorado.edu/es/simulation/ fraction-matcher. Los cambios que se evidencian en la actualidad abarcan prácticamente todas las actividades humanas, desde lo político, económico y social hasta, ciertamente, la educación. Este contexto variable interviene en la 60 forma y estilo de vida de los individuos que conforman la sociedad, y de manera especial a los jóvenes y adolescentes.
Una de las alternativas que existen en la actualidad es la utilización de la tecnología, que ofrece una gama de herramientas para disímiles actividades de la vida diaria. Estas facilitan una serie de medios que se pueden valer para el trabajo docente y como estrategia de enseñanza-aprendizaje para el estudiante.

El presente estudio estableció la incidencia del uso del simulador Phet en el aprendizaje de las fracciones equivalentes de los estudiantes de octavo grado de bachillerato en Matemáticas, de la Institución Educativa General Santander. Arbeláez (2010) menciona que la utilización de simulación y de videojuegos se ha transformado en los últimos años en una poderosa herramienta de formación. Lo anterior lo complementa Aguirre (2012) cuando explica que la simulación se ha generalizado en los últimos 20 años en la formación de los estudiantes del mundo, debido a la necesidad que tienen los docentes de utilizar esta herramienta didáctica.

En esta investigación se trabajó específicamente en el simulador Phet en lenguaje Java, aplicable a las fracciones equivalentes. De los resultados de esta investigación se puede aseverar que el uso del simulador Java incide en el aprendizaje de las fracciones equivalentes y se observa la diferencia significativa entre los datos estadísticos de los grupos 1 y 2 , como resultado de la incidencia en uso de simuladores en el desarrollo de la unidad didáctica entre ambos grupos. 
Arias (2009) resalta que la simulación puede utilizarse como un recurso para la formación de nuevos conceptos y para la adquisición de nuevos conocimientos, destrezas, estrategias y comportamiento. En un estudio realizado por Méndez (2014) se afirma que "el uso de simuladores Java mejora el aprendizaje del teorema del trabajo-energía entre el pretest y postest de los estudiantes de cuarto bachillerato" (p. 58). (Díaz, 2016), Evidenció que el aplicativo Phet como herramienta virtual de aprendizaje mejoró el rendimiento académico significativamente al utilizar la simulación en la enseñanza de las fracciones equivalentes. Como recomendación queda abierta la eventualidad de continuar con la exploración de los diferentes niveles de dificultad que ofrece la plataforma Phet, de la Universidad de Colorado, respecto al tema de las fracciones, de analizar y evaluar el comportamiento de cada estudiante con el uso del simulador y poderlo integrar al contexto escolar de la Institución Educativa General Santander, por ser considerada esta plataforma un recurso pedagógico puesto a disposición de los docentes de matemáticas, y que se pueda adaptar y formalizar en el aula de clase.

\section{Conclusiones}

A partir del análisis estadístico de los datos aplicando la prueba t student, se puede observar que no hay evidencia para rechazar la hipótesis nula; por lo tanto, las medias son estadísticamente iguales. Es decir, no se aprecian diferencias representativas entre la media del pretest y del postest del grupo 1 evaluado. Esto significa que el rendimiento académico de los estudiantes de octavo grado para el grupo 1 no mejoró de manera significativa al utilizar el simulador Phet en la enseñanza de las fracciones equivalentes.

Ahora, a partir del análisis estadístico de los datos aplicando la prueba t student, se puede observar que hay evidencia para rechazar la hipótesis nula; por consiguiente, las medias son diferentes. En este caso, la media del postest es estadísticamente mayor que la media del pretest del grupo 2. Es decir que el rendimiento académico de los estudiantes de grado octavo mejoró de manera notable para el grupo 2, al utilizar el simulador Phet en la enseñanza de las fracciones equivalentes.

\section{Referencias}

Aguirre, G. (2012). Postura experiencial de los docentes que utilizan la simulación clínica como estrategia didáctica en la carrera de Medicina. (Tesis de Maestría). Universidad Nacional de Colombia. Recuperado de http://www.bdigital. unal.edu.co/7716/1/4868234.20 12.pdf

Arbeláez, M. (2010). Mundos virtuales para la educación en salud simulación y aprendizaje en Open Simulator (Tesis de Maestría). Recuperada de http:// www.maestriaendiseno.com/ pdf/ mauricioarbelaez.pdf 
Arias, L. (2009). La simulación computarizada en el proceso de enseñanza aprendizaje de electrónica. Recuperado el 16 de abril de 2016, de http://www.ilustrados.com/ tema/3575/simulacion-computarizadaproceso-ensenanza-aprendizajeElectronica.html.

Bagur, A. (2011). Matemáticas para todos. Recuperado el 16 de abril de 2016, de http://www.acmor.org.mx/descargas/ mate106.pdf

Balestrini, M. (2006). Cómo se elabora el proyecto de investigación. Recuperado el 16 de abril de 2016, de http://dip. una.edu.ve/mae/metodologiali/paginas/ Balestrini,\%20M\%20Cap\%20VI\%20 U2.pdf

Díaz, J. (2016). Aplicación Phet: estrategia de enseñanza aprendizaje de fracciones equivalentes. Revista Criterios, 23(1), 111-123.

Durán, E. (2012). Red de tecnología educativa. Recuperado de http:// reddetecnologiaeducativa.bligoo.com. co/aprendiendo-matematicas-con-laayuda-de-simuladores

Fatela, M. (2012). Simuladores. Recuperado el 16 de abril de 2016, de http://www. fatela.com.ar/PaginasWeb/simuladores. htm

Galicia, A. (2005). Las aulas virtuales en el proceso enseñanza aprendizaje en el nivel medio, ciclo básico, sector privado del área urbana del municipio de Jalapa, departamento de Jalapa. (Tesis de Licenciatura). Recuperado de http://biblioteca.usac.edu.gt/ 62 tesis/07/07_1713.pdf

García, D. (2012). Promover en el aula estrategias de aprendizaje para elevar el nivel escolar de los alumnos de tercero de primaria en el área de matemáticas (Tesis de Licenciatura). Recuperada de http:// biblio3. url.edu.gt/Tesis/2012/05/24/ Garcia-Daniela.pdf

Huapaya, G. (2012). Modelación usando función cuadrática: experimentos de enseñanza con estudiantes de 5to de secundaria. Lima-Perù.

Huillcañahui, M. (2010). Muestreo aleatorio simple. Recuperado el 14 de diciembre de 2014, de http://www.slideshare.net/ milit/muestreo-aleatorio-simple

López, A. (2011). Prueba de normalidad. Recuperado de http://es.slideshare. net/leugimxw/pruebas-de-normalidadprueba-de-andersondarling

Monje, C. (2011). Metodología de la investigación cuantitativa y cualitativa. Guía didáctica. Recuperado de https:// carmonje.wikispaces.com/file/view/ Monje+Carlos+Arturo+-+Gu\%C3\% ADa+did\%C3\%Alctica+Metodolog $\%$ C3\%ADa+de+la+investigaci\%C3 \%B3n.pdf

Ortega, M. (2001). Sistemas de ineteracción persona-computador. Castilla-La Mancha: Ediciones de la Universidad de CastillaLa Mancha.

Pósito, R. (2012). El problema de enseñar y aprender ciencias naturales en los nuevos ambientes educativos. (Tesis de Maestría). Recuperada de http://sedici.unlp.edu. ar/bitstream/handle/10915/18190/ Documento_completo.pdf?sequence=3

Pérez, C. (2011). Fisim: simulador físicomatemático integrado a la plataforma de gestión del aprendizaje zera. Recuperado el 16 de abril de 2016, de: https://www.researchgate.net/ 
publication/229068441_FISIM_ SIMULADOR_FISICO_-_MATEMATICO_ INTEGRADO_A_LA_PLATAFORMA_DE_ GESTION_DEL_APRENDIZAJE_ZERA

Peña, P. y Alemán, A. (2013). Teoría de simuladores. Recuperado de http:// www.aves.edu.co/ovaunicor/recursos/1/
index_Simulacion_por_computador. pdf

Shuttleworth, M (2008). Diseño de la investigación cuantitativa. Recuperado el 25 de febrero de 2016, de https:// explorable.com/es/diseno-de-lainvestigacion-cuantitativa 